\title{
The Predictive Value of Initial CT Scan in Patients with COVID-19
}

\author{
Tahir Belice ${ }^{1,{ }^{*}}$, Nejla Ozkan Yıldırım ${ }^{1}$, Ozden Yildirim Akan $^{1}$ and Arif Yuksel ${ }^{1}$ \\ ${ }^{1}$ Department of Internal Diseases, University of Health Science, Izmir Bozyaka Training and Research Hospital, Izmir, Turkey \\ "Corresponding author: Department of Internal Diseases, University of Health Science, Izmir Bozyaka Training and Research Hospital, Karabaglar Mah 485 Sk, Izmir, Turkey. Tel: \\ 00905062817530, Fax: 00902322505050,Email: drtahirelf@hotmail.com
}

Received 2020 July 13; Revised 2020 November 30; Accepted 2020 December 02.

\begin{abstract}
Background: As the pandemic of coronavirus disease 2019 (COVID-19) in Wuhan raised public health concerns, many studies were conducted to reveal the risk factors of the disease and predictive prognostic indicators to plan the treatment strategies and commence therapies earlier. Although specific parameters, including age, gender, chronic diseases, D-dimer, and neutrophillymphocyte-ratio play a curial role in COVID-19, the association between mortality and the extent of lung involvement in the initial computerized tomography (CT) scan of patients diagnosed with COVID-19 has not been well documented in the literature.

Objectives: We compared two groups (CT-1 and CT-2) in terms of lung involvement in the initial CT of patients admitted to the emergency unit and then hospitallzed with COVID-19.

Methods: Using the digital data system of İzmir Bozyaka Training and Research Hospital, Turkey, subjects diagnosed with COVID-19 in April 2020 were retrospectively screened. For each of the 90 patients, the initial CT scans on admission were evaluated for the extent of lung involvement and classified as CT-1 ( $\leq 5 \%$ of lung involvement, n: 45$)$ and CT-2 (> 5\% of lung involvement, n: 45 ).

Results: There was not any statistical significance between two different CT groups regarding mortality and length of stay of patients in hospital with COVID-19 $(\mathrm{P}=0.72$ and $\mathrm{P}=0.51$, respectively). Neutrophil counts were found to be statistically significant and higher in CT-2 group than the CT-1 $(\mathrm{P}=0.02)$ group. The length of stay in hospital was correlated with age, neutrophil-lymphocyte ratio (NLR), neutrophils, lymphocyte, and only the correlation between length of stay in hospital and age was significant $(\mathrm{P}=0.01)$. Conclusions: The size of involvement is not a predictive indicator for mortality and length of stay in hospital in patients with COVID-19.
\end{abstract}

Keywords: COVID-19, Neutrophils, Tomography, X-Ray Computed Tomography

\section{Background}

A novel coronavirus with a characteristic crown morphology at scanning electron microscopy, causing coronavirus disease 2019 (COVID-19) that is spread by humanto-human transmission due to close contacts, was identified in China and announced by the World Health Organization (WHO) as a pandemic on Mar 11, 2020 (1). No specific antiviral drugs are available to treat COVID-19; thus, early detection and adherence to medical isolation are crucial (2). To date, computerized tomography (CT) images are used by physicians for a fast and effective diagnosis of COVID-19 (3). Based on the literature, patchy groundglass opacities with interlobular septal and vascular thickening, consolidative opacities with a rounded pattern, and a peripheral distribution are the basic findings of COVID-19 in chest CT (4). These findings are helpful to the radiologist in the early detection and diagnosis of patients. Early disease recognition can speed up treatments and prompt rapid patient isolation (5). Chest CT cannot alone fully ex- clude the diagnosis of COVID-19, particularly in the early stage of the infection $(3,4)$. Because of the prodromal phase of COVID-19, viral infection manifests with symptoms before the emergence of imaging manifestations (6). A normal chest CT scan does not exclude the diagnosis, and also CT findings can vary as pneumonia progresses $(4,5)$. The data of CT findings for COVID-19 in the literature indicate similar features to the other coronaviruses (severe acute respiratory syndrome(SARS) and the Middle East respiratory syndrome (MERS) (7). The diagnosis of COVID19 with CT scan is more sensitive than the initial reverse transcription-polymerase chain reaction (RT-PCR) and is also relatively simple and quick (8). Because of its invasiveness and objectivity, CT scan is also used to follow up patients with COVID-19. Although the chest CT examination plays a crucial role in the initial diagnosis of COVID-19, the relationship between the initial CT findings, including the extent of the involvement, and mortality and length of stay in hospital has not well been documented yet.

The neutrophil-lymphocyte ratio (NLR) was known as 
an indicator of inflammatory status, and a higher level of NLR in a patient with COVID-19 has been proved as a risk factor of mortality $(9,10)$. The negative outcomes of COVID-19 were found to be associated with a higher NLR, mostly an NLR of $>3.13$ (11). Thus, the higher NLR levels were known as an indicator of the severity of COVID-19 as well as a hyperinflammatory state (11). However, the counts of neutrophil, NLR, and lymphocyte have not been studied in detail to compare the extent of lung involvement in CT. Furthermore, the association of mortality with the extent of lung involvement in the initial CTs of patients diagnosed with COVID-19 has not well been documented in the literature.

\section{Objectives}

Finding the answer to the question of whether the size of infected areas in the lung is associated with mortality and length of stay in hospital in patients with COVID-19 is essential. The present study aimed at revealing whether the extent of COVID-19 lung involvement in CT could predict the disease aggravation and assessing the association between the extent of lung involvement and the mortality and length of stay in hospital.

\section{Methods}

\subsection{Data Collection and Study Design}

Patients diagnosed with COVID-19 were retrospectively screened using the digital data system of İzmir Bozyaka Training and Research Hospital, Turkey. Inclusion criteria were the age of 18 years or more and hospital admission with COVID-19 from April 1, 2020, to April 30, 2020. G-Power (ver. 3.1.9.4) software was used to estimate the sample size considering a type 1 of $5 \%$ error and test power of $85 \%$. Using random sampling, the participants were selected from the hospital data system in April 2020. The exclusion criterion was the subjects who did not meet the inclusion criteria. Radiologists reported all initial CT images as the percentage of lung involvement during admission in the pandemic office of the emergency unit. The time interval from disease to CT was the same for all the cases, and it was reported during the admission phase. For each of the 90 patients, the initial CT scans on admission were evaluated for the percentage of lung involvement, and then we classified the patients as CT- 1 ( $\leq 5 \%$ of lung involvement, $n=45$ ) and CT-2 (over $5 \%$ of lung involvement, $n=45$ ).

\subsection{Statistical Analysis}

An independent t-test and one-way analysis of variance (ANOVA) were employed to compare the variables.
The results were presented using descriptive statistics; mean \pm standard deviation (SD) for continuous variables, and the number and percentage for categorical variables. For numeric variables, first, the normal distribution of the data was tested using the one-sample KolmogorovSmirnov test, and according to the results of this test, the results of numeric variables were described using mean \pm $\mathrm{SD}$, minimum, and maximum. For the statistical analysis, the Statistical Package for the Social Sciences (SPSS) software (IBM SPSS for Windows, ver.24) was used. P-values of $<0.05$ were considered statistically significant.

\section{Results}

\subsection{Patients}

A total of 90 patients with $49(54.4 \%)$ men $(M=60.9$ years, $S D=16.1)$ and $41(45.6 \%)$ women $(M=66.9, S D=17.8)$ were included in the present study. The mean age was 66.76 years $(S D=19.10)$ in the CT-1 group and 61.67 years $(S D=$ $14.97)$ in the CT-2 group. The mean age between the two groups was not statistically significant $(P=0.16)$. When the patients were compared in terms of gender, no statistical significance was found between length of stay in hospital, mortality, and lung involvement in CT between men and women $(\mathrm{P}=0.14, \mathrm{P}=0,78$, and $\mathrm{P}=0.67$, respectively). When mortality rates were compared with the parameters, including neutrophils, NLR, lymphocyte using an independent t-test, a statistically significant difference was found between mortality rates and parameters, including neutrophils and NLR $(\mathrm{P}=0.06$ and $\mathrm{P}=0.02$, respectively). In other words, as NLR and neutrophil values increased, mortality rates increased. The length of stay in hospital was correlated with age, NLR, neutrophils, and lymphocyte, but only the age was positively correlated with length of stay in hospital $(\mathrm{P}=0.00)$.

\subsection{The Extent of Lung Involvement and the Length of Stay in Hospital}

The length of stay in hospital was 8.24 days ( $S D=1.083$ ) in the CT-1 group and 7.34 days $(S D=0.832)$ in the CT-2 group. The mean length of stay between the two groups was not statistically significant $(P=0.51)$.

\subsection{The Extent of Lung Involvement and Mortality}

The total number of fatal cases was 9 (\% 10), including six men (12.2\%) and three women (7.3\%). CT-1 and CT-2 groups were compared with the Chi-square test for mortality, and the percentage of fatal cases was $8.9 \%(n=4)$ in the CT-1 group and 11.1\% $(n=5)$ in the CT-2 group. However, no statistically significant difference was found between the extent of lung involvement and mortality $(\mathrm{P}=0.72)$. 
4.4. Comparison the Counts of the Neutrophil and Lymphocytes, and NLR with the Extent of Lung Involvement

The following results were found in the CT-1 group: NLR: 5.7 (SD = 9.82), neutrophil: $5.06 \times 10^{3} / \mu \mathrm{L}(\mathrm{SD}=3.13)$, and lymphocyte: $1.61 \times 10^{3} / \mu \mathrm{L}(\mathrm{SD}=2.61)$, whereas they were as follows in the CT-2 group: NLR: 6.6 (SD = 8.82), neutrophil: $7.52 \times 10^{3} / \mu \mathrm{L}(\mathrm{SD}=6.49)$, and lymphocyte: 1.62 $\times 10^{3} / \mu \mathrm{L}(\mathrm{SD}=0.83)$. We compared CT-1 and CT-2 groups for NLR, neutrophil, and lymphocyte. Only neutrophil levels were found with a statistically significant difference between both groups. In other words, neutrophil counts were statistically higher in the CT-2 group $(\mathrm{P}=0.02)$.

\section{Discussion}

In the early days of the emergence of COVID-19, most of the cases were determined to report exposure to food from the Huanan Seafood Wholesale Market (12). The pathogenesis of COVID-19 is still not wholly understood, and cytokine storm is thought to play crucial roles in disease severity (13). However, around 81\% of patients with COVID-19 develop a mild type of disease, and they never need hospitalization (14). The severity of COVID-19 varies from mild to severe, and it cannot be easily predicted using a specific tool or criteria beforehand. However, in several studies, some of the parameters have been shown to be associated with mortality and length of stay in hospital in patients with COVID-19. In a study on 191 patients diagnosed with COVID-19 from China, the most crucial risk factors were age, having higher sequential organ failure assessment (SOFA) scores, and D-dimer of over $1 \mu \mathrm{g} / \mathrm{mL}$, and these findings could give physicians an idea about poor prognosis at an early stage (15). In 338 patients diagnosed with COVID-19 in New York from March 10 - 26, 2020, a chest x-ray severity score was predictive of risk for hospital admission and intubation (16). Wang et al. revealed that C-reactive protein (CRP) could be a predictive marker in COVID-19, and its threshold value was $26.9 \mathrm{mg} / \mathrm{L}$ to anticipate the possibility of aggravation (17). In the present study, we compared two CT groups that were separated based on the extent of lung involvement (less than $5 \%$ or over) in patients with COVID-19, and we aimed at revealing whether there was a significant difference in mortality and length of stay in hospital between two groups. There were no statistically significant differences in these parameters from between CT-1 and CT-2 groups (t-test; $\mathrm{P}=0.517$; Chi-square test, $\mathrm{P}=$ $0.725)$. We could not find another study comparing the extent of lung involvement in the initial CT of patients with COVID-19. Thus, we hope that these findings can be valuable for further studies.

Viruses can trigger NETosis, the process of neutrophil extracellular traps (NETs) generation, and several diseases, including venous thromboembolism, cancer, diabetes, and infections involved in NETosis (18). Virus-induced NETs can generate inflammation in an uncontrolled way (19). The effect of COVID-19 on both venous and arterial thromboembolism has been shown in many studies $(15,20)$. The relationship between thromboembolism and COVID-19 is a crucial factor regarding fatal cases because NETosis was proved to trigger the development of thromboembolism $(21,22)$. In the present study, the parameters of NLR, lymphocyte, and neutrophil were compared both in the CT-1 and CT-2 groups, and only a statistically significant difference was found in neutrophil that was higher in the CT-2 group $(\mathrm{P}=0.02)$. Even though the neutrophil count was higher in the CT-2 group, the mortality and length of stay in the hospital showed no significant difference between the two CT groups. However, in all participants, as the age and parameters, including NLR and neutrophil, increased, mortality rates increased significantly $(P<0.05)$. These findings can be an answer to the question of why the mortality is not related to the extent of lung involvement in initial CT of patients with COVID-19, but the NLR and neutrophil are associated. The most crucial reason for fatal cases might be associated with NETosis, but not the extent of involvement. Therefore, the initial CTs of patients with COVID-19 would not be a predictor of mortality. We need more data to find a rapid tool for the diagnosis and progression of the disease, including machine learning or artificial intelligence other than CT, clinical, or laboratory findings (23).

This study had several limitations. First, the study included only one center with a small sample, and no patient had a follow-up chest CT after admission. Second, only 90 patients diagnosed clinically and radiologically were included; negative results or other viruses were not included in the analyses. Also, we did not consider the final survival outcome, and the exact causes of death were not known.

\subsection{Conclusion}

Patients diagnosed with COVID-19 need to be evaluated as soon as possible, and the prognosis should be predicted based on specific parameters, including NLR, gender, age, etc. The extent of lung involvement in COVID-19 was not associated with mortality in the present study, but we need to explore the unknown features of COVID-19 with large samples and further studies.

\section{Acknowledgments}

Special thanks to all co-workers that collaborated with us in this study from X Hospital. 


\section{Footnotes}

Authors' Contribution: NOY,TB, and OYA designed the study. AY, TB, NOY, and OYA drafted the manuscript AY, TB, NOY, and OYA analyzed the data. AY,TB, and OYA revised the manuscript.

\section{Conflict of Interests: None.}

Ethical Approval: The study was approved by the Bozyaka Training and Research Hospital Ethics Committee (Date:21.05.2020, Number:15345988-193-2020).

\section{Funding/Support: None.}

Informed Consent: All the procedures in the study involving the human subjects were conducted in accordance with the ethical standards of the institutional research committee and with the 1964 Helsinki declaration and its later amendments or comparable ethical standards.

\section{References}

1. Ren LL, Wang YM, Wu ZQ, Xiang ZC, Guo L, Xu T, et al. Identification of a novel coronavirus causing severe pneumonia in human: a descriptive study. Chin Med J (Engl). 2020;133(9):1015-24. doi: 10.1097/CM9.0000000000000722. [PubMed: 32004165]. [PubMed Central: PMC7147275].

2. Wu R, Wang L, Kuo HD, Shannar A, Peter R, Chou PJ, et al. An update on current therapeutic drugs treating COVID-19. Curr Pharmacol Rep. 2020:1-15. doi: 10.1007/s40495-020-00216-7. [PubMed: 32395418]. [PubMed Central: PMC7211915].

3. Abbasi-Oshaghi E, Mirzaei F, Farahani F, Khodadadi I, Tayebinia H. Diagnosis and treatment of coronavirus disease 2019 (COVID-19): Laboratory, PCR, and chest CT imaging findings. Int J Surg. 2020;79:143-53. doi:10.1016/j.ijsu.2020.05.018. [PubMed:32422384]. [PubMed Central: PMC7227548]

4. Xiong Y, Sun D, Liu Y, Fan Y, Zhao L, Li X, et al. Clinical and highresolution ct features of the COVID-19 infection: Comparison of the initial and follow-up changes. Invest Radiol. 2020;55(6):332-9. doi:10.1097/RLI.0000000000000674. [PubMed:32134800]. [PubMed Central: PMC7147282]

5. Dai WC, Zhang HW, Yu J, Xu HJ, Chen H, Luo SP, et al. CT imaging and differential diagnosis of COVID-19. Can Assoc Radiol J. 2020;71(2):195200. doi: 10.1177/0846537120913033. [PubMed: 32129670]. [PubMed Central: PMC7140975].

6. Wang K, Kang S, Tian R, Zhang X, Zhang X, Wang Y. Imaging manifestations and diagnostic value of chest CT of coronavirus disease 2019 (COVID-19) in the Xiaogan area. Clin Radiol. 2020;75(5):341-7. doi: 10.1016/j.crad.2020.03.004. [PubMed: 32216961]. [PubMed Central: PMC7118637].

7. Petrosillo N, Viceconte G, Ergonul O, Ippolito G, Petersen E. COVID19, SARS and MERS: are they closely related? Clin Microbiol Infect. 2020;26(6):729-34. doi: 10.1016/j.cmi.2020.03.026. [PubMed: 32234451]. [PubMed Central: PMC7176926].

8. He JL, Luo L, Luo ZD, Lyu JX, Ng MY, Shen XP, et al. Diagnostic performance between CT and initial real-time RT-PCR for clinically suspected 2019 coronavirus disease (COVID-19) patients outside Wuhan, China. Respir Med. 2020;168:105980. doi: 10.1016/j.rmed.2020.105980. [PubMed: 32364959]. [PubMed Central: PMC7172864].

9. Faria SS, Fernandes PJ, Silva MJ, Lima VC, Fontes W, Freitas-Junior R, et al. The neutrophil-to-lymphocyte ratio: a narrative review. Ecancermedicalscience. 2016;10:702. doi: 10.3332/ecancer.2016.702. [PubMed: 28105073]. [PubMed Central: PMC5221645].
10. Qin C, Zhou L, Hu Z, Zhang S, Yang S, Tao Y, et al. Dysregulation of immune response in patients with coronavirus 2019 (COVID-19) in Wuhan, China. Clin Infect Dis. 2020;71(15):762-8. doi: 10.1093/cid/ciaa248. [PubMed: 32161940]. [PubMed Central: PMC7108125].

11. Liu J, Liu Y, Xiang P, Pu L, Xiong H, Li C, et al. Neutrophil-to-lymphocyte ratio predicts severe illness patients with 2019 novel coronavirus in the early stage. medRxiv. 2020;2020. doi: 10.1101/2020.02.10.20021584.

12. Wu C, Chen X, Cai Y, Xia J, Zhou X, Xu S, et al. Risk factors associated with acute respiratory distress syndrome and death in patients with coronavirus disease 2019 pneumonia in Wuhan, China. JAMA Intern Med. 2020;180(7):934-43. doi: 10.1001/jamainternmed.2020.0994. [PubMed: 32167524]. [PubMed Central: PMC7070509].

13. Channappanavar R, Perlman S. Pathogenic human coronavirus infections: causes and consequences of cytokine storm and immunopathology. Semin Immunopathol. 2017;39(5):529-39. doi: 10.1007/s00281-017-0629-x. [PubMed: 28466096]. [PubMed Central: PMC7079893].

14. Wu Z, McGoogan JM. Characteristics of and Important Lessons From the Coronavirus Disease 2019 (COVID-19) Outbreak in China: Summary of a Report of 72314 Cases From the Chinese Center for Disease Control and Prevention. JAMA. 2020;323(13):1239-42. doi 10.1001/jama.2020.2648. [PubMed: 32091533].

15. Zhou F, Yu T, Du R, Fan G, Liu Y, Liu Z, et al. Clinical course and risk factors for mortality of adult inpatients with COVID-19 in Wuhan, China: a retrospective cohort study. Lancet. 2020;395(10229):105462. doi: 10.1016/S0140-6736(20)30566-3. [PubMed: 32171076]. [PubMed Central: PMC7270627].

16. Toussie D, Voutsinas N, Finkelstein M, Cedillo MA, Manna S, Maron SZ, et al. Clinical and chest radiography features determine patient outcomes in young and middle-aged adults with COVID-19. Radiology. 2020;297(1):E197-206. doi: 10.1148/radiol.2020201754. [PubMed: 32407255]. [PubMed Central: PMC7507999].

17. Wang G, Wu C, Zhang Q, Wu F, Yu B, Lv J, et al. C-reactive protein level may predict the risk of COVID-19 aggravation. Open Forum Infect Dis. 2020;7(5):ofaa153. doi: 10.1093/ofid/ofaa153. [PubMed: 32455147]. [PubMed Central: PMC7197542].

18. Muraro SP, De Souza GF, Gallo SW, Da Silva BK, De Oliveira SD, Vinolo MAR, et al. Respiratory Syncytial Virus induces the classical ROS dependent NETosis through PAD-4 and necroptosis pathways activation. Sci Rep. 2018;8(1):14166. doi: 10.1038/s41598-018-32576-y. [PubMed: 30242250]. [PubMed Central: PMC6154957].

19. Schonrich G, Raftery MJ. Neutrophil extracellular traps go viral Front Immunol. 2016;7:366. doi: 10.3389/fimmu.2016.00366. [PubMed: 27698656]. [PubMed Central: PMC5027205].

20. Klok FA, Kruip M, van der Meer NJM, Arbous MS, Gommers D Kant KM, et al. Incidence of thrombotic complications in critically ill ICU patients with COVID-19. Thromb Res. 2020;191:145-7. doi 10.1016/j.thromres.2020.04.013. [PubMed: 32291094]. [PubMed Central: PMC7146714].

21. Fuchs TA, Brill A, Duerschmied D, Schatzberg D, Monestier M, Myers DJ, et al. Extracellular DNA traps promote thrombosis. Proc Natl Acad SciUSA.2010;107(36):15880-5. doi:10.1073/pnas.1005743107. [PubMed: 20798043]. [PubMed Central: PMC2936604].

22. Borissoff JI, Joosen IA, Versteylen MO, Brill A, Fuchs TA, Savchenko AS, et al. Elevated levels of circulating DNA and chromatin are independently associated with severe coronary atherosclerosis and a prothrombotic state. Arterioscler Thromb Vasc Biol. 2013;33(8):2032-40. doi: 10.1161/ATVBAHA.113.301627. [PubMed: 23818485]. [PubMed Central: PMC3806482]

23. El Homsi M, Chung M, Bernheim A, Jacobi A, King MJ, Lewis S, et al. Review of chest CT manifestations of COVID-19 infection. Eur J Radiol Open. 2020;7:100239. doi: 10.1016/j.ejro.2020.100239. [PubMed: 32550256]. [PubMed Central: PMC7276000]. 